\title{
Enabling Secondary Access through Robust Primary User Channel Assignment
}

\author{
Jad Nasreddine, Andreas Achtzehn, Janne Riihijärvi, Petri Mähönen \\ Institute for Networked Systems, RWTH Aachen University \\ Kackertstrasse 9, D-52072 Aachen, Germany \\ Email: $\{$ jad, aac, jar, pma $\} @$ inets.rwth-aachen.de
}

\begin{abstract}
A common concept to mitigate the effects of spectrum scarcity is to allow opportunistic dynamic spectrum access (DSA) to licensed frequency bands. In this context, the license holder may experience a decrease in the performance of its network. For cellular networks this may lead to unwanted coverage holes at locations where the secondary-induced interference is too strong. In this paper we propose a planning tool and a channel assignment mechanism for cellular OFDMA-based networks that takes the coverage requirements of the primary system into account. By keeping a dynamic reserve of channels for those nodes that experience the highest interference, we can maximize spectrum utilization while guaranteeing a predefined service reliability. Our simulations show that only a small fraction of channels will need exclusive reservation, hence large parts of the spectrum may be employed in secondary systems.

Index Terms-Dynamic Spectrum Access; OFDMA; coverage reduction; channel assignment, planning.
\end{abstract}

\section{INTRODUCTION}

Recent studies and measurement campaigns have revealed the significant under-utilization of precious spectrum resources due to static frequency allocation regimes [1]. A new paradigm, Dynamic Spectrum Access (DSA), has emerged as a solution to these spectrum inefficiency problems by allowing a more flexible spectrum assignment [2], [3].

In the context of DSA, different concepts were considered. One of these concepts allows opportunistic access of the secondary users to licensed spectrum. In order to access the primary spectrum, secondary users should guarantee that they will not disrupt primary communications. In the last decade, most of the research work was focusing on secondary network resource management and primary detection. However, only few papers consider, though implicitly, the definition of primary constraints and the development of mechanisms to allow secondary activity [4]-[8]. Most of these works assume that the primary license holder is a TV broadcasting network and that the activity of any secondary network is enabled by a reduction in geographical coverage, with the exception of [8] where a reduction of rate is considered in cellular networks.

The main objective of cellular operators is to serve their users with their requested services wherever they are. This means that a pervasive coverage, especially in urban zones, has high priority in operator decisions. Therefore, a cellular operator will allow opportunistic access to its licensed spectrum only if its pervasive coverage constraint is satisfied. By coverage, we mean here the geographic area where the signals of a transmitter can be decoded by the receiver. In other words, cellular operators will not allow secondary activity if it will lead to the appearance of "dead zones" in their coverage, where the mobile cannot make reliable communications. In this paper, we propose a solution to this problem that does not lead to a noticeable loss in coverage or data rate. This will be achieved by exploiting the flexibility of OFDMA-based systems, which are the majority of next generation wireless networks (e.g. 3GPP LTE, IEEE 802.16 and IEEE 802.11n), and performing intelligent planning and channel assignment mechanisms. The proposed solution will lead to more robust primary networks against secondary interference for the same available secondary opportunities, or more opportunities for the same robustness in comparison to other solutions.

The paper is organized as follows. In Section II we formulate the problem and describe the system model. In Section III we describe the proposed approach, which is basically composed of a planning tool and a channel assignment algorithm. In Section IV we present the simulation model and discuss the obtained results. Finally, we conclude the paper in Section V.

\section{Problem Formulation and System Model}

We shall focus here on a scenario where the primary network is an OFDMA-based network with $T$ frequency channels. We assume that all channels are orthogonal and show the same propagation characteristics. We also assume that the secondary network will respect the interference constraint of the primary network that can be reflected by a maximum allowed interference $\iota_{\max }$. This interference is determined such that it can be tolerated by any primary receiver, especially the ones at the cell boundaries [9]. In addition, we assume that any approximation in the computation should lead to a more protective situation for the primary network, guaranteeing that, in the worst case, the performance of this network is the one predicted by the model. Finally, we assume that all base stations have the same activity pattern that is defined by the probability of activity $\alpha$, which is the ratio between the number of active users in the cell and $T$ (i.e. $\alpha$ reflects the cell load).

The dimensioning of cellular networks is designed to guarantee that the probability of having an unsatisfied user is $\varepsilon$. An unsatisfied user can appear due to coverage or cell capacity problems. In most cases, users at the edges of the cell are the ones that are most affected by these problems. Therefore network planning tools are designed to guarantee that the 
average Signal to Interference and Noise Ratio (SINR) at the edges of the cell is higher than a given threshold $\gamma_{\min }$. The average SINR in $\mathrm{dB}$ at the edge of the cell is given by

$$
\gamma_{\text {edge }}=P-L_{\max }-10 \log \left(10^{I_{S, \max } / 10}+10^{P_{N} / 10}\right),
$$

where $P$ is the transmitted power by the base station for one user, $L_{\max }$ is the maximum allowed path loss in the cell, $I_{S, \max }$ is the interference generated by the other cells in the primary network and $P_{N}$ is the power of background noise.

When a secondary user is allowed to transmit, additional interference will be experienced by the primary users, and the minimum SINR becomes

$$
\begin{aligned}
\gamma_{s}= & P-L_{\max } \\
& -10 \log \left(10^{I_{S, \max } / 10}+10^{P_{N} / 10}+10^{\iota / 10}\right),
\end{aligned}
$$

where $\iota$ is the experienced interference due to secondary transmission. Therefore, all primary users will experience a reduction in their SINRs if no action is taken by the network (e.g. power control, change of modulation). Due to the planning of wireless networks, a user at cell boundary is normally served with the most efficient configuration (e.g. highest power) and hence it does not support additional interference. Thus, the primary network has to accept an increase in the number of its unsatisfied users to allow secondary users to communicate. One commonly used method is to compromise the coverage area of the primary cell and reduce its cell radius from $r$ to $r_{s}$. If the secondary network exploits all the opportunities and the primary network keeps its normal coverage planning (i.e., without considering secondary activity), "dead zones" where primary users cannot have reliable communication will appear in the system. However, dead zones are unacceptable in most cellular systems. Thus, the primary network will either increase the transmit power of its equipments, including user equipments, or will have to add more base stations in the area. Both solutions are costly and thus should be avoided.

In order to maintain the same base station distribution and configuration while reducing the impact of secondary activity on the coverage area, the primary network can use a more sophisticated planning combined with a channel assignment algorithm guaranteeing that a high percentage of users in the otherwise dead zone will be able to maintain reliable communication. Therefore, we propose to divide the cell into two regions as shown in Fig. 1, depicted as "protected zone" and "inner zone", and allow secondary transmission only in channels that are allocated to users in the inner zone. The protected zone is the zone where the interference generated by the secondary nodes will lead to primary users' SINRs lower than $\gamma_{\min }$ in contrary to the inner zone where the primary users will always have an SINR higher than $\gamma_{\min }$. The border between the two zones is defined by the threshold where the SINRs $\gamma_{s}$ of the users are equal to $\gamma_{\min }$. In order to allow user equipment to detect in which zone they are, an estimation of the SINR has to be performed. However, since the interference from the secondary network cannot be distinguished from the other determinants of interference, the receiver estimating its

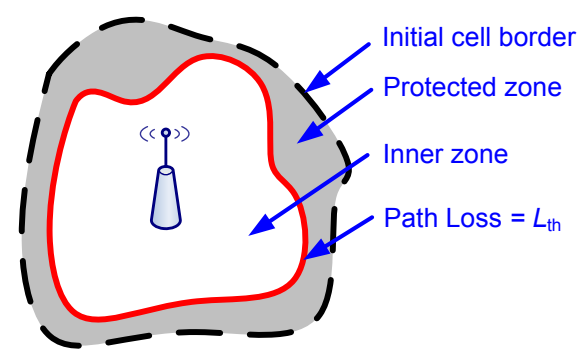

Fig. 1. Protected and inner zones in a primary cell separated by $L_{\text {th }}$.

SINR will not be able to know if this SINR already includes secondary interference. Therefore, the primary network has to employ another method to identify the position of the receiver inside the cell by measuring the path loss towards its serving base station. Hence, the border between the two zones can be now defined by the zone where the path loss is equal to threshold $L_{\text {th }}$. In our model, the primary network will divide its channels into two groups: Shared channels can be used by the secondary users in addition to primary users in the inner zone whereas restricted channels can be only used by primary users in the protected zone and if required for the primary users in the inner zone. The primary network will associate $\tau$ restricted channels from the $T$ channels for primary use only with priority to users in the protected zone. This will guarantee a full coverage of the primary network while allowing the users in the protected zone to have SINRs higher than $\gamma_{\min }$. In this way, the users at the initial cell border will experience the same SINR as was expected in the planning phase since there is no secondary users' interference, while the users at the new border, defined by $L=L_{\text {th }}$, will also have SINRs higher than $\gamma_{\min }$ since this zone is defined to support an additional interference that can reach $\iota_{\max }$. This value is used by the secondary network in order to estimate its allowed power and tune its detection mechanisms [9], [10]. The SINR at the initial cell border without secondary activity should be equal to the SINR at the inner zone border with secondary activity. From (1) and (2), we can write

$$
\begin{aligned}
L_{\mathrm{th}}= & L_{\max }+10 \log \left(10^{I_{S, \max } / 10}+10^{P_{N} / 10}\right) \\
& -10 \log \left(10^{I_{S, t h} / 10}+10^{P_{N} / 10}+10^{\iota_{\max } / 10}\right),
\end{aligned}
$$

where $I_{S, t h}$ is the estimated internal interference experienced by the users at the border of the inner zone. $L_{\max }$ and $I_{S, \max }$ are in general given by the planning tool and depend on the deployment of the base stations.

In the following we shall propose mechanisms to compute $L_{\text {th }}$ and, as our main contribution, find the maximum number of shared channels while guaranteeing unsatisfied user probability due to secondary activity lower than $\varepsilon_{s}$, where $\varepsilon_{s}<\varepsilon$. This probability can be also defined as the probability of having at least one user from the protected zone associated to a shared channel:

$$
\varepsilon_{s}=\mathbb{P}\left\{\exists(i \in \Pi \text { and } f \in \Theta) \text { such that } a_{i f}=1\right\},
$$


where $a_{i f} \in\{0,1\}$ is a variable that reflects the binding of channel $f$ to user $i$ (i.e., it is equal to 1 if channel $f$ is assigned to user $i$ and 0 otherwise), $\Pi$ is the set of users in the protected zone and $\Theta$ is the set of shared channels.

The proposed channel assignment is assumed to be perfect, meaning that at any time we can reassign channels in order to preserve availability of the restricted channels to users in the protected zone. More practical algorithms and their performance can be derived from the following analysis but are out of the scope of this paper.

\section{Planning And Channel Assignment MECHANISMS}

In this section, we first propose a simple method to compute the path loss threshold and a method to estimate the number of channels that should be reserved as restricted channel. This computation will be normally performed during the planning phase and may also be performed periodically based on changes in the network configuration. Then, we describe a simple channel assignment.

\section{A. Computation of Path Loss Threshold}

The path loss threshold $L_{\text {th }}$ is computed to identify those users in the protected zones: if $L>L_{\text {th }}$ the user is considered to be in the protected zone. The value of $L_{\text {th }}$ can be computed from (3), where the only unknown parameter is $I_{S, t h}$ that does not only depend on the position of the primary user inside the cell but also on the distribution of the base stations and their activity. Therefore, the value of $L_{\text {th }}$ will depend on the scenario. Since we adopt a protective scheme for the primary network, we assume that $I_{\mathrm{S}, \mathrm{th}}=10 \log (\alpha)+I_{S, \max }$. The parameter $\alpha$ is introduced in order to take the impact of cell loads into account. This is not optimal for increasing spectrum opportunities but it will guarantee that the primary users will always be satisfied. By using this assumption, we can write

$$
\begin{aligned}
L_{\mathrm{th}}= & L_{\max } \\
& +10 \log \left(\frac{10^{I_{S, \max } / 10}+10^{P_{N} / 10}}{\alpha 10^{I_{S, \text { max }} / 10}+10^{P_{N} / 10}+10^{\iota_{\max } / 10}}\right) .
\end{aligned}
$$

\section{B. Computation of the Number of Shared Frequencies}

We shall now carry out some analytical calculations on the distribution of path loss for the different users in the typical primary cell. Our objective is to derive expressions for the probability that a typical user experiences path loss greater than $L_{\text {th }}$, and from that derive the probability that the given number of restricted channels is sufficient for all primary nodes in the cell.

We assume that the locations of users in the cell are independent and identically distributed, with the p.d.f. of the distance from the base station denoted by $p_{r}$. Further, we assume that the users are distributed isotropically around the base station, allowing us to focus only on the radial component of the location distribution. This assumption is without loss of generality. For a user at distance $r$ (in meters) from the base station, we write the path loss in $\mathrm{dB}$ as

$$
L=10 \eta \log r+C+\chi,
$$

where $C$ and $\eta$ are constants specific to the propagation environment, and $\chi$ is a normal random variable of zero mean and standard deviation $\sigma$ to account for shadowing. Thus, the probability that the user is assigned a restricted channel is

$$
\mathbb{P}\left\{L>L_{\text {th }}\right\}=1-\mathbb{P}\left\{10 \eta \log r+C+\chi \leq L_{\text {th }}\right\} .
$$

We shall denote by $L_{r} \equiv 10 \eta \log r+C$ the first component of the path loss. Since $r$ is a random variable, $L_{r}$ is also random, and we shall indicate its p.d.f. by $p_{L_{r}}$. Explicit expression for $p_{L_{r}}$ can be obtained from $p_{r}$ by the standard formula for the p.d.f. of the transform of a random variable (see, for example, [11] for details). The probability density function $p_{L}$ of the total path loss is then given by the convolution of the densities of the two components, that is, by

$$
p_{L}(t)=\int_{-\infty}^{\infty} p_{L_{r}}(s) p_{\chi}(s-t) d s,
$$

where

$$
p_{\chi}(x)=\frac{1}{\sqrt{2 \pi}} \exp \left(-\frac{x^{2}}{2 \sigma^{2}}\right) .
$$

Depending on the exact radial distance distribution $p_{r}$ of the users, (8) can either be evaluated analytically in closed form, or by numerical techniques such as multiplying the fast Fourier transforms (FFTs) of $p_{L_{r}}$ and $p_{\chi}$, and taking the inverse FFT of the result.

Now, given $p_{L}$, we can obtain the probability that the path loss of a typical user exceeds $L_{\text {th }}$ as

$$
P_{\mathrm{th}} \equiv \mathbb{P}\left\{L>L_{\mathrm{th}}\right\}=1-\int_{-\infty}^{L_{\mathrm{th}}} p_{L}(t) d t .
$$

Since we assume the locations and the shadow fading components of the path losses of the different users to be i.i.d., the number of users $N_{p}$ in the protected zone is given by the binomial distribution with parameter $P_{\mathrm{th}}$. In particular, the probability that sufficient number of protected channels have been reserved is given by

$$
\begin{aligned}
\mathbb{P}\left\{N_{p} \leq \tau\right\} & =\sum_{i=0}^{\tau}\left(\begin{array}{c}
N \\
i
\end{array}\right) P_{\mathrm{th}}^{i}\left(1-P_{\mathrm{th}}\right)^{N-i} \\
& =(N-\tau)\left(\begin{array}{c}
N \\
\tau
\end{array}\right) \int_{0}^{1-P_{\mathrm{th}}} t^{N-\tau-1}(1-t)^{\tau} d t,
\end{aligned}
$$

where $N$ is the total number of users in the cell. This probability is proportional to the regularized incomplete beta function $I_{1-P_{\mathrm{th}}}(N-\tau, \tau+1)$ which can be evaluated numerically [12]. In order to satisfy primary constraints, this probability should be greater or equal to $1-\varepsilon_{s}$. Therefore, the planning tool will find the number of channels $\tau$ that satisfies

$$
\mathbb{P}\left\{N_{p} \leq \tau-1\right\} \leq 1-\varepsilon_{s} \leq \mathbb{P}\left\{N_{p} \leq \tau\right\} .
$$


The planning tool will compute the value of $\tau$ using (12). As shown in this sub-section, the value of $\tau$ depends also on the total number of users $N$ in the cell. Moreover, the value of $\tau$ depends on $L_{\text {th }}$ that is related to $\iota_{\max }$ and $N$, which is reflected by $\alpha$, as shown in sub-section III-A. Therefore, the planning tool will provide the channel assignment algorithm with a table that gives the number of restricted channels $\tau$ as a function of $N$ and $\iota_{\max }$.

Since the value of $N$ can change on a millisecond scale, it is not practical to use instantaneous values of $N$. Instead, the planning tool will provide the channel assignment with a table that gives the total number of users $N$ as a function of the period of day, the day in the week, etc. The value of $N$ will be estimated as the maximum number of users that the cell can have at a given period of time since we are adopting conservative mechanisms to protect primary networks. Another possibility is to consider $N$ equal to the maximum number of users in the cell over all time periods. However, this will limit the opportunities for the secondary network since in some periods of time the cell load can be very low (e.g., cells in an industrial zone will be less loaded at night or at the weekend).

\section{Channel Assignment}

The primary network will reserve $\tau$ channels for the users in the protected zone based on the period of time and the table provided by the planning tool. Each time a primary user from this zone requests a connection, the primary network will try to assign a restricted channel. If there is no restricted channel available and some of these channels are allocated to users in the inner zone, one of these channels will be allocated to the new user and a shared channel will be allocated to the user that was originally associated to the restricted channel. Otherwise, a shared channel will be associated to the user until a restricted channel is released. Moreover, if the system does not have any available channel from the $T$ channels, the user will be dropped.

For each period of time, the primary network will inform the secondary network about channels that can be used for secondary communications and the value of $\iota_{\max }$ that is needed to compute the maximum allowed power. In order to compute this power, the secondary needs to know the boundaries inside which the primary users in the shared channels are. In theory, this should be the circle that contains the inner zone [9]. Since this depends on the shadowing factor which follows a normal distribution, we can estimate the radius $r_{s}$ of this cell by considering the tail of the distribution. Another more conservative approach is to assume that it is equal to the radius of the cell that contains the full coverage area of the cell, which is the approach that we have taken in this paper.

\section{Simulation And Results}

We evaluate the performance of the proposed mechanism using Xia-Bertoni propagation model [13]. We assume that the central frequency is $2 \mathrm{GHz}$. Therefore, the values of $C$ and $\eta$
TABLE I

SIMULATION PARAMETERS.

\begin{tabular}{ll}
\hline System parameter & value \\
\hline Bandwidth $W$ & $10 \mathrm{MHz}$ \\
Number of channels $T$ & 50 \\
Cell radius $r$ & $500 \mathrm{~m}$ \\
Path loss threshold $L_{\max }$ & $128.65 \mathrm{~dB}$ \\
Noise power $P_{N}$ & $-121 \mathrm{dBm}$ \\
Transmitted power per channel $P$ & $29 \mathrm{dBm}$ \\
Internal interference $I_{S, \max }$ & $-110 \mathrm{dBm}$ \\
\hline
\end{tabular}

are $15.65 \mathrm{~dB}$ and 3.76, respectively. Simulation parameters are summarized in Table I.

We consider two types of user distribution inside the cell. In the first one, the users are uniformly distributed inside a cell of radius $r$. In the second type, the distance between the user and the base station follows a truncated Gaussian distribution between 0 and $r$ with mean 0 and standard deviation $r / 3$. The second type of distribution corresponds roughly to the case of microcells or hotspots [14].

We shall first study the variation of the number of restricted channels as a function of cell load $\alpha$ and probability $\varepsilon_{s}$ for a fixed value of $\iota_{\max }$, which is depicted in Fig. 2. As expected, $\tau$ is an increasing function of $\alpha$ and a decreasing function of $\varepsilon_{s}$ since an increase in $\alpha$ will lead to an decrease in $L_{\text {th }}$ (see (5)) which will increase the number of users in the protected zone, whereas an increase in $\varepsilon_{s}$ will lead to a less protective scenario for the primary network allowing more opportunities to the secondary. It should be noted here that the proposed mechanism allows the secondary network to use more than $70 \%$ of its channels even when the system is fully loaded and with an allowed interference $\iota_{\max }$ that is $21 \mathrm{~dB}$ higher than the noise power. We note that the value of $L_{\mathrm{th}}$ varies only in the interval $[118.54 \mathrm{~dB}, 118.94 \mathrm{~dB}]$ when $\alpha$ decreases from 1 to 0.02 . It is also clear that the number of restricted channels are much lower when we have the Gaussian distribution because the users will be mainly concentrated around the base station. In this case the shared channels can reach $95 \%$ of the primary channels when the primary network is fully loaded.

In Fig. 3, we show the variation of $\tau$ as a function of the maximum allowed interference $\iota_{\max }$ and the probability $\varepsilon_{s}$ for a fully loaded cell. First, we note that the value of $L_{\text {th }}$ varies in the interval $[69 \mathrm{~dB}, 128.65 \mathrm{~dB}]$ when $\iota_{\max }$ varies between $-150 \mathrm{dBm}$ and $-60 \mathrm{dBm}$. When $\iota_{\max }$ reaches $-120 \mathrm{dBm}$ the value of $L_{\text {th }}$ becomes $128 \mathrm{~dB}$, which is equal to $L_{\max }$ and therefore it stabilizes at this value for the lower values of $\iota_{\max }$. Fig. 3 shows that there is a limit of $\iota_{\max }$ (i.e. $-75 \mathrm{dBm}$ for the uniform distribution and $-40 \mathrm{dBm}$ for the the Gaussian distribution) over which no channels can be shared. Moreover the figure shows that the proposed algorithm can allow sharing more than $90 \%$ of primary channels when it is fully loaded and for $\iota_{\max }$ lower than $-120 \mathrm{dBm}$.

Table II is the configuration table that will provided by the planning tool for $\varepsilon_{s}=0.05$. This table gives the number of restricted channels as a function of $\iota_{\max }$ and $\alpha$. If the primary network knows the range of its load at a given period, it can 


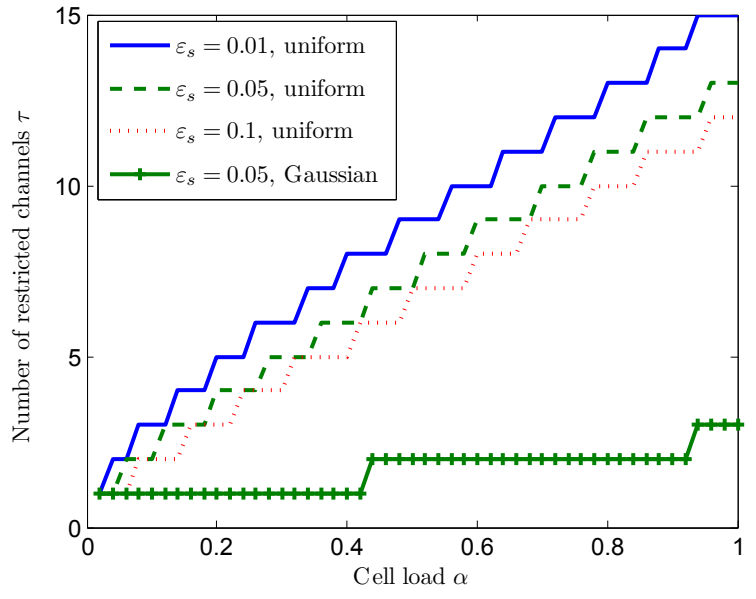

Fig. 2. The variation of the number of restricted channels $\tau$ as a function of the cell load $\alpha$ for different values of $\varepsilon_{s}$ and $\iota_{\max }=-100 \mathrm{dBm}$.

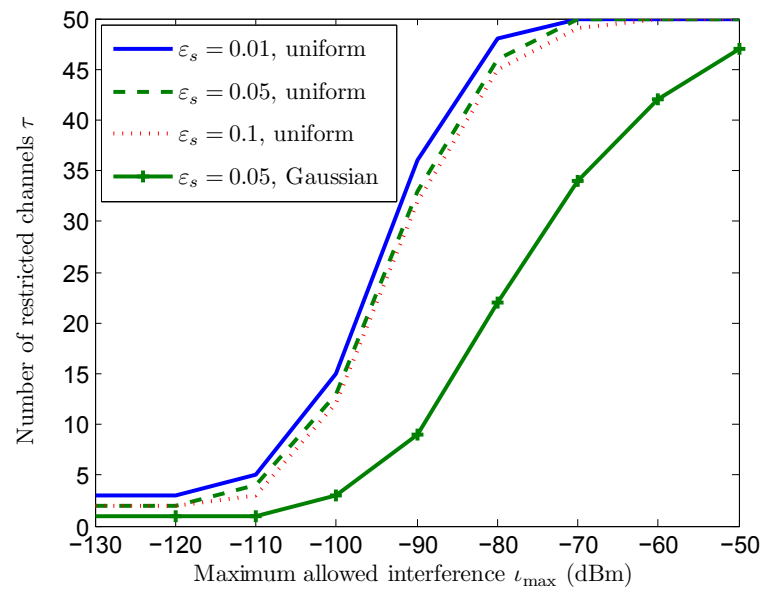

Fig. 3. The variation of the number of restricted channels $\tau$ as a function of the maximum allowed interference $\iota_{\max }$ for different values of $\varepsilon_{s}$ and a fully loaded cell (i. e., for $\alpha=1$ ).

choose the highest bound in the table for a given $\iota_{\max }$. For instance, if $\iota_{\max }$ is $-90 \mathrm{dBm}$ and the load changes between 0.3 and 0.5 at night time, the primary network will reserve 9 restricted channels for uniform distribution and 2 channels for Gaussian distribution.

\section{CONCLUSiOnS}

In this paper we have designed a planning tool to estimate the number of channels an OFDMA-based primary system must reserve for exclusive use in order to keep its quality-ofservice requirements. From the derivation of the probability of primary users to face interference above the system-dependent tolerable threshold we were able to provide upper boundaries on the overall system performance.

The measurements we have encountered in our simulations are promising: With the proposed reservation scheme a significant amount of spectrum still remain available for secondary sharing. The algorithms developed are lightweight, yet for practical deployments may be replaced by an even simpler
TABLE II

CONFIGURATION TABLE FOR $\varepsilon_{s}=0.05$ FOR THE UNIFORM (THE FIRST NUMBER IN THE PARENTHESIS) AND THE GAUSSIAN (THE SECOND NUMBER IN THE PARENTHESIS) USER DISTRIBUTIONS.

\begin{tabular}{cccccc}
\hline & \multicolumn{5}{c}{$\iota_{\max }$} \\
\cline { 2 - 6 }$\alpha$ & $-130 \mathrm{dBm}$ & $-110 \mathrm{dBm}$ & $-90 \mathrm{dBm}$ & $-70 \mathrm{dBm}$ & $-50 \mathrm{dBm}$ \\
\hline 0.2 & $(1,1)$ & $(1,1)$ & $(4,1)$ & $(10,6)$ & $(10,10)$ \\
0.4 & $(1,1)$ & $(1,1)$ & $(6,1)$ & $(19,10)$ & $(20,18)$ \\
0.6 & $(1,1)$ & $(1,1)$ & $(9,2)$ & $(28,14)$ & $(30,26)$ \\
0.8 & $(2,1)$ & $(2,1)$ & $(11,2)$ & $(37,18)$ & $(40,34)$ \\
1 & $(2,1)$ & $(2,1)$ & $(13,3)$ & $(46,22)$ & $(50,42)$ \\
\hline
\end{tabular}

lookup table. The approach we have taken is flexible enough to account for many different deployment scenarios.

\section{ACKNOWLEDGMENT}

We acknowledge a partial financial support from European Union through ARAGORN and FARAMIR projects. We also thank the financial support from Deutsche Forschungsgemeinschaft and RWTH Aachen University through UMIC-research centre. We would also like to thank Huawei Technologies Co., Ltd. for providing partial funding of this work through the CIRION project.

\section{REFERENCES}

[1] "Spectrum policy task force report," Federal Communications Commission ET Docket 02-135, November 42008.

[2] E. Hossain, D. Niyato, and Z. Han, Dynamic Spectrum Access and Management in Cognitive Radio Networks. Cambridge University Press, 2009

[3] I. F. Akyildiz, W.-Y. Lee, M. C. Vuran, and S. Mohanty, "Next generation/dynamic spectrum access/cognitive radio wireless networks: a survey," Computer Networks, vol. 50, no. 13, pp. 2127 - 2159, 2006.

[4] E. G. Larsson and M. Skoglund, "Cognitive radio in a frequencyplanned environment: some basic limits," IEEE Transactions on Wireless Communications, vol. 7, no. 12, pp. 4800-4806, December 2008.

[5] N. Hoven and A. Sahai, "Power scaling for cognitive radio," in the International Conference on Wireless Networks, Communications and Mobile Computing, vol. 1, June 2005, pp. 250-255.

[6] M. Vu, N. Devroye, and V. Tarokh, "The primary exclusive region in cognitive networks," in the 5th IEEE Consumer Communications and Networking Conference (CCNC 2008), Jan. 2008, pp. 1014-1019.

[7] A. Sahai, R. Tandra, S. M. Mishra, and N. Hoven, "Fundamental design tradeoffs in cognitive radio systems," in the first international workshop on Technology and policy for accessing spectrum (TAPAS 2006). New York, NY, USA: ACM, 2006.

[8] S. Srinivasa and S. A. Jafar, "How much spectrum sharing is optimal in cognitive radio networks?" IEEE Transactions on Wireless Networks, vol. 7, no. 10, pp. 4010 - 4018, October 2008.

[9] J. Nasreddine, J. Riihijärvi, and P. Mähönen, "Location-based adaptive detection threshold for dynamic spectrum access," in the 4th IEEE International Symposium on New Frontiers in Dynamic Spectrum Access Networks (DySPAN 2010), Singapore, April 2010.

[10] J. Nasreddine, O. Sallent, J. Pérez-Romero, and R. Agustí, "Positioningbased framework for secondary spectrum usage," Physical Communication, vol. 1, no. 2, pp. 121-133, 2008.

[11] G. Grimmett and D. Stirzaker, Probability and random processes. Oxford University Press, USA, 2001.

[12] W. H. Press, B. P. Flannery, S. A. Teukolsky, and W. T. Vetterling., Numerical Recipes, Second, Ed. Cambridge University Press, 1992.

[13] L. Maciel, H. Bertoni, and H. Xia, "Unified approach to prediction of propagation over buildings forall ranges of base station antenna height," IEEE transactions on vehicular technology, vol. 42 , no. 1, pp. 41-45, 1993.

[14] R. K. Ganti and M. Haenggi, "Interference and outage in clustered wireless ad hoc networks," IEEE Transaction on Information Theory, vol. 55, pp. 4067-4086, Spetember 2009. 